Correspondence

Marwan M. Azar

marwan.azar@yale.edu

Received 16 February 2015 Accepted 19 March 2015

\section{Cervicofacial necrotizing fasciitis caused by Haemophilus influenzae serotype e in a patient with Sjogren's syndrome, systemic lupus erythematosus and hypocomplementaemia}

\author{
Marwan M. Azar, ${ }^{1}$ David Folk, ${ }^{2}$ Triona Henderson, ${ }^{3}$ Saral Mehra, ${ }^{2}$ \\ Eugenia Vining ${ }^{2}$ and David B. Banach ${ }^{1}$ \\ ${ }^{1}$ Section of Infectious Diseases, Yale School of Medicine, 333 Cedars Street, PO Box 208022, 25 \\ New Haven, CT 06520-8022, USA \\ ${ }^{2}$ Section of Otolaryngology Head and Neck Surgery, Yale School of Medicine, 333 Cedars Street, \\ PO Box 208022, 25 New Haven, CT 06520-8022, USA \\ ${ }^{3}$ Department of Pathology, Yale School of Medicine, PO Box 208022, 25 New Haven, \\ CT 06520-8022, USA
}

\begin{abstract}
Introduction: Haemophilus influenzae has the potential to lead to invasive disease including necrotizing fasciitis, especially in immunocompromised individuals. There are only eight previous reports of monomicrobial necrotizing fasciitis due to this organism, and, to the best of our knowledge, none of the head and neck. There is also limited epidemiological and clinical data on infections caused by non-b serotypes of $H$. influenzae, including serotype e.
\end{abstract}

Case presentation: A 60-year-old female, with systemic lupus erythematosus, Sjogren's syndrome and hypocomplementaemia, presented with rapidly progressive right-sided facial and neck pain, swelling and erythema. She was treated initially with broad-spectrum antibiotics and underwent emergent debridement of the infected tissues. Intraoperative cultures and blood cultures grew $H$. influenzae. She required two additional debridements and was treated with ceftriaxone for 3 weeks with resolution of her symptoms.

Conclusion: Here, we present the first reported case, to the best of our knowledge, of cervical necrotizing fasciitis due to $H$. influenzae serotype e. This case highlights the continued clinical importance of $H$. influenzae as a cause of invasive disease, including necrotizing fasciitis, particularly in immunocompromised individuals. Moreover, the case provides an example of invasive $H$. influenzae infection caused by a non-b serotype, an emerging concern in the era of widespread $H$. influenzae serotype b vaccination.

Keywords: cervicofacial; Hemophilus influenzae; necrotizing fasciitis; serotype e.

\section{Introduction}

Haemophilus influenzae is an aerobic Gram-negative coccobacillus with broad pathogenic potential found in the upper respiratory tract. Although it principally causes respiratory infections such as rhinosinusitis, otitis media, bronchitis and pneumonia, $H$. influenzae can lead to invasive disease, including meningitis, bacteraemia and necrotizing fasciitis. Whilst most cases of necrotizing fasciitis are polymicrobial, monomicrobial necrotizing fasciitis does

Abbreviations: CT, computed tomography; Hia, Haemophilus influenzae serotype a; Hib, $H$. influenzae serotype b; Hie, $H$. influenzae serotype e; Hif, $H$. influenzae serotype $f$; SLE, systemic lupus erythematosus.. occur but is rarely caused by bacteria other than Streptoccocus or Staphylococcus spp. There are only eight previously reported cases of monomicrobial necrotizing fasciitis due to $H$. influenzae. Here, we describe the first reported case, to the best of our knowledge, of cervicofacial necrotizing fasciitis with $H$. influenzae serotype e (Hie), occurring in an immunocompromised patient, including her presentation, diagnosis and outcome. We also review all eight previously reported cases of necrotizing fasciitis caused by $H$. influenzae, including the serotyping results. This case highlights the importance of the immune system, especially the role of complement, in the pathogenesis of invasive disease with $H$. influenzae, as well as providing an example of invasive infection caused by a non-b serotype, an emerging 
concern in the era of widespread vaccination against $H$. influenzae serotype $\mathrm{b}(\mathrm{Hib})$.

\section{Case report}

A 60-year-old woman with a history of Sjogren's syndrome, leukocytoclastic vasculitis and systemic lupus erythematosus (SLE) presented to our emergency department with new-onset right-sided facial pain and swelling. She first noticed right pre-auricular pain upon awakening the previous day. Subsequently, her pain intensified and spread laterally to involve her right periorbital and maxillary areas and caudally to her right mandible and upper neck. She also developed erythema and worsening oedema of the affected areas, as well as subjective fevers and chills, prompting a visit to the hospital for evaluation. Her past medical history was notable for Sjogren's syndrome and SLE with associated hypocomplementaemia $\left(\mathrm{C} 3,62 \mathrm{mg} \mathrm{dl}^{-1} ; \mathrm{C} 4,12 \mathrm{mg} \mathrm{dl}^{-1}\right)$, leukocytoclastic vasculitis and Raynaud's syndrome for which she was previously receiving mycophenolate mofetil $1000 \mathrm{mg}$ twice daily, and hydroxychloroquine $200 \mathrm{mg}$ daily and had received an infusion of rituximab $1000 \mathrm{mg}$ 3 months prior to this presentation. Two years prior to admission, she had a right submandibular lymph node excision and open biopsy of the right submandibular gland for persistent tender adenopathy. Pathology was consistent with inflammation and negative for Sjogren's syndrome.

In the emergency department, she appeared ill. She denied visual disturbances and difficulty breathing or swallowing. Her temperature was $103^{\circ} \mathrm{F}$, blood pressure was $187 / 67 \mathrm{mmHg}$, pulse was 103 beats $\mathrm{min}^{-1}$, respiratory rate was 12 breaths $\min ^{-1}$ and oxygen saturation was $95 \%$ while breathing ambient air. Examination of the face showed erythema, tenderness and oedema of the right periorbital area leading to partial closure of the eye, right cheek, mandible and upper neck, without palpable crepitus. The skin of the upper face and upper chest did not appear to be involved. Her extra-ocular movements, pupillary reflexes and cranial nerves were intact. Oral cavity examination showed no purulence from Stensen's duct and no obvious signs of pharyngitis. Laboratory analysis was notable for leukocytosis with a neutrophil predominance (white blood cell count of $17000 \mathrm{~mm}^{-3}$ with $90 \%$ neutrophils). Intravenous clindamycin was administered and a flexible laryngoscopy was performed, revealing an oedematous and friable right buccal mucosa with a normal appearance of the nasopharynx, vocal cords and epiglottis.

Upon re-evaluation $6 \mathrm{~h}$ later, the patient's overall appearance had worsened, with marked lip swelling and complete closure of the right eye, as well as new erythema over the upper chest and development of dysphagia. A repeat flexible laryngoscopy showed excoriated mucosa in the nasal canal, new oedema of the palate, uvula and right aryepiglottic fold, and partial obstruction of the right vocal cord. Vancomycin, ceftazidime and metronidazole were administered intravenously in addition to dexamethasone for laryngeal oedema. A computed tomography (CT) scan with intravenous contrast showed significant oedema of the subcutaneous tissues and deep fascial layers surrounding the right parotid, masticator and carotid spaces extending to the mid-neck (Fig. 1). The patient was taken urgently to the operating room where she underwent fibre-optic intubation, tracheostomy and direct laryngoscopy, followed by right deep neck exploration and debridement. Turbid 'dishwater' fluid was encountered immediately subcutaneously. No dental source of infection was identified. Intraoperative findings were notable for a slimy biofilm and diffuse murky fluid within the fascial planes of the neck and parapharyngeal space without frank purulence. All neck planes and levels were dissected including the carotid sheath, retropharyngeal space and parapharyngeal space, as well as excision of multiple enlarged jugulodigastric lymph nodes. Of note, with subplatysmal flap elevation, a mature scar in the submandibular region completely dehisced from destruction of this tissue, but all of the cervical skin itself was viable and left intact. A pulsed irrigator with 21 saline admixed with $900 \mathrm{mg}$ clindamycin solution was used to irrigate the entire deep neck and parapharyngeal space, and necrotic fascial tissue was debrided down to healthy bleeding muscle with preservation of all major neurovascular structures. A number of Penrose drains were placed, including deep into the parapharyngeal and retropharyngeal spaces, and the neck was packed with moist gauze and left open for

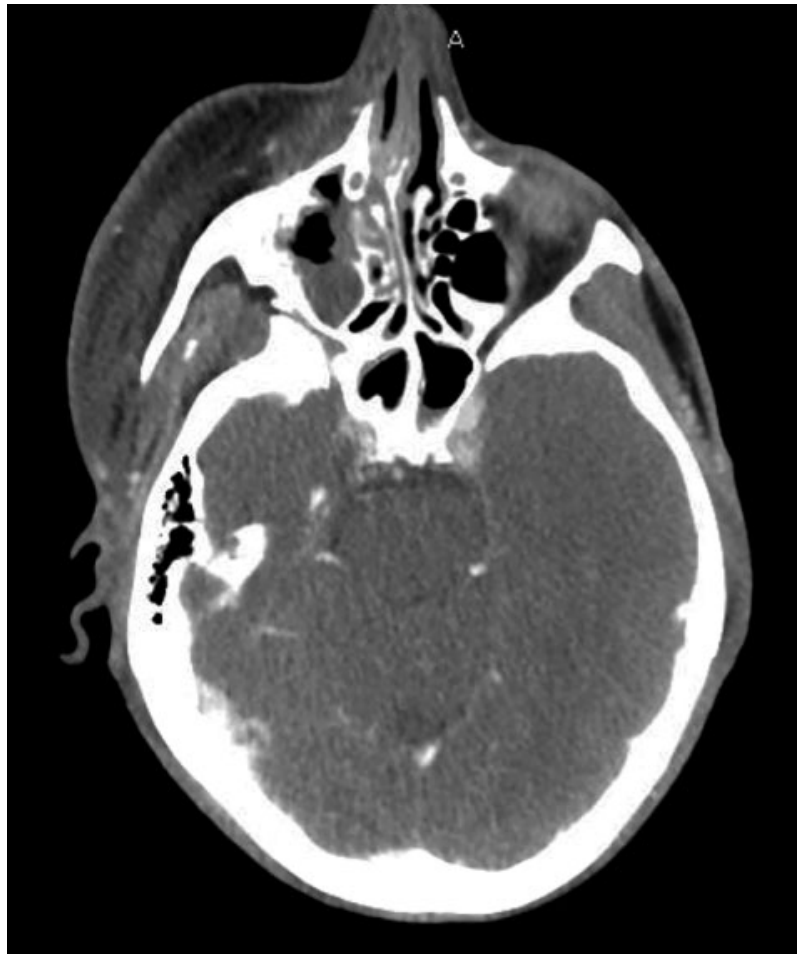

Fig. 1. CT of the head and neck with contrast showing extensive soft-tissue oedema involving the right parotid, masticator and carotid spaces extending from the upper neck down to the deep right tonsillar fossa and right retropharyngeal space. 
twice-daily packing changes. A CT scan of the chest with contrast was performed due to the risk of mediastinal extension via the danger space and did not reveal findings suggestive of mediastinitis. Surgical site cultures from the necrotic fascia and the aerobic bottles of two sets of blood cultures obtained on admission grew H. influenzae (Fig. 2). Chromogenic $\beta$-lactamase enzyme testing was performed and was negative, and the isolate was reported as sensitive to ampicillin. Antibiotics were narrowed to ceftriaxone $2 \mathrm{~g}$ daily given the patient's history of a rash with use of amoxicillin-clavulanate. The patient underwent two additional deep neck explorations for debridement and washout, followed by staged closure of the neck wound over a Penrose drain 4 days after the initial procedure. She improved and was discharged 7 days after admission without a tracheotomy tube with plans to complete a 3-week course of ceftriaxone. The patient reported receiving her vaccinations as a child but could not recall whether she received the full Hib vaccination series. The $H$. influenzae isolate was determined to be serotype e by slide agglutination testing using commercially available antisera for each of the serotypes a-f, at the Connecticut Department of Public Health laboratory. She was seen in the outpatient infectious disease clinic 4 weeks after discharge and reported complete resolution of her symptoms. Immunoglobulin levels obtained 8 weeks after discharge were normal to elevated (IgM, $260 \mathrm{mg} \mathrm{dl}^{-1}$; IgA, $212 \mathrm{mg} \mathrm{dl}^{-1}$; IgG, total $2330 \mathrm{mg} \mathrm{dl}^{-1}$; IgG1, $1500 \mathrm{mg} \mathrm{dl}^{-1}$; IgG2, $254 \mathrm{mg} \mathrm{dl}^{-1}$; IgG3, $179 \mathrm{mg}$ $\mathrm{dl}^{-1}$; IgG4, $16.1 \mathrm{mg} \mathrm{dl}^{-1}$ ). In addition, antibodies to Hib were checked and were equal to $3.9 \mu \mathrm{g} \mathrm{ml}^{-1}$, suggestive of protection against this serotype (levels $>1.0 \mu \mathrm{g} \mathrm{ml}^{-1}$ are considered protective).

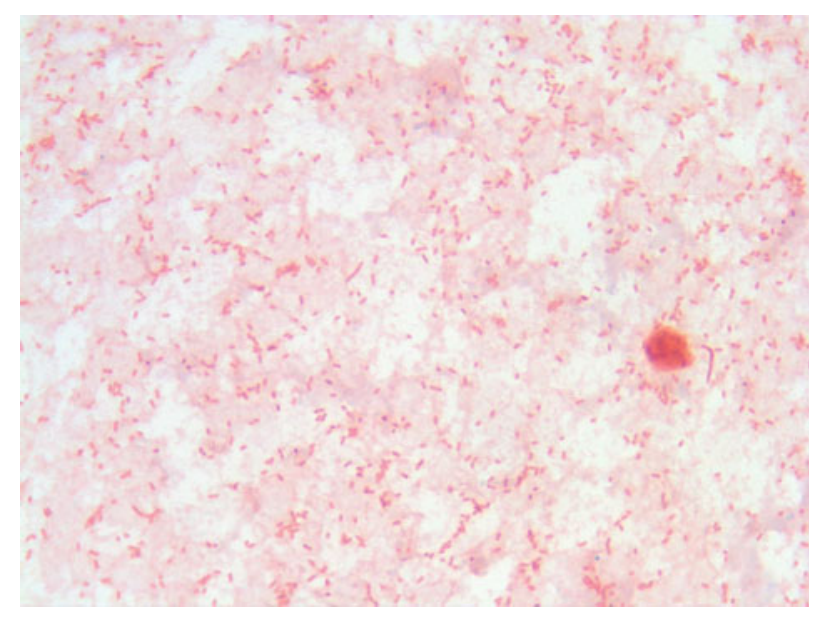

Fig. 2. Gram stain from a blood culture bottle showing numerous Gram-negative coccobacilli.

\section{Discussion}

Necrotizing fasciitis is most often a polymicrobial process (type 1) in which anaerobic bacteria are isolated in combination with facultative aerobes. Monomicrobial (type 2) necrotizing fasciitis occurs less commonly, and is most often caused by streptococci. There are only eight published accounts of monomicrobial necrotizing fasciitis due to H. influenzae (Table 1) in the literature (Chalmers, 2010; Collette et al., 1987; Lee \& Ip, 2010; McLellan et al., 2008; Robinson et al., 2010; Saito et al., 2009; Stumvoll \& Fritsche, 1997; Ugai et al., 2014). To the best of our knowledge, this is the first report of monomicrobial cervicofacial necrotizing fasciitis due to $H$. influenzae. In a retrospective study of 45 patients with cervicofacial necrotizing fasciitis, $H$. influenzae was isolated from operative cultures in one case, although it is unclear whether other organisms were grown concomitantly (Mathieu et al., 1995). Almost half of the cases included in the study reported a predisposing condition including diabetes, alcoholism and malignancy. In an analysis of all cases of craniocervical necrotizing fasciitis over an 11-year period at one institution, all cases were polymicrobial in nature and none was due to $H$. influenzae (Bahu et al., 2001). It is well known that patients with compromised immune systems, including complement and immunoglobulin deficiencies, are at increased risk for invasive infections with $H$. influenzae (Martinot et al., 2014; Winkelstein \& Moxon, 1992). In the majority of the published case reports of necrotizing fasciitis with $H$. influenzae, a predisposing condition including chemotherapy, use of immunomodulating medications or diabetes mellitus was present. Our patient was treated previously with several immunosuppressive medications for SLE and Sjogren's syndrome. Moreover, she had documented hypocomplementaemia.

Among its many functions in host defence, the complement system plays a particular role in eliminating encapsulated bacteria. Available evidence suggests that both the classic and alternative pathways are activated in infection with H. influenzae (Winkelstein \& Moxon, 1992). In individuals with $\mathrm{C} 2$ and $\mathrm{C} 4$ deficiency, the classic complement pathway is interrupted, but as the alternative pathway remains functional, patients are at only moderately increased risk of infection. In contrast, C3 deficiency hampers both branches of the complement cascade, leading to significantly increased risk of infection with $H$. influenzae (Tedesco, 2008). Interestingly, patients with C5-C9 deficiency are not more susceptible to infection than the general population (Tedesco, 2008). This suggests that the opsonic activity of $\mathrm{C} 3$ is more central to the host's defence against $H$. influenzae than the bactericidal activity of C5-C9 (Winkelstein \& Moxon, 1992). Robinson et al. (2010) described the case of a 17-year-old female with SLE on immunosuppressive therapy who presented with simultaneous right arm and leg necrotizing fasciitis. As in our case, this patient's marked hypocomplementaemia may have played a role in her disease process.

The importance of opsonization is also exhibited by the humoral immune system's role in killing of $H$. influenzae. 
Table 1. Reported cases of necrotizing fasciitis secondary to $H$. influenzae

\begin{tabular}{|c|c|c|c|c|c|}
\hline Study & $\begin{array}{l}\text { Age, sex and location of } \\
\text { necrotizing fasciitis }\end{array}$ & Predisposing condition(s) & $\begin{array}{l}\text { Source of culture } \\
\text { positivity }\end{array}$ & $\begin{array}{l}\text { H. influenzae } \\
\text { serotype }\end{array}$ & $\begin{array}{l}\text { Antibiotic duration } \\
\text { and outcome }\end{array}$ \\
\hline Present case & $\begin{array}{l}\text { 60-year-old female; right } \\
\text { mandible, neck and face }\end{array}$ & $\begin{array}{l}\text { Sjogren's syndrome; SLE on } \\
\text { mycophenolate and } \\
\text { hydroxychloroquine; } \\
\text { hypocomplementaemia }\end{array}$ & Surgical tissues, Blood & Type e & $\begin{array}{l}\text { Ceftriaxone for } 3 \text { weeks; multiple debridements } \\
\quad(n=3) \text {; recovery }\end{array}$ \\
\hline Ugai et al. (2014) & $\begin{array}{l}\text { 59-year-old female; right } \\
\text { thigh }\end{array}$ & $\begin{array}{l}\text { Colon cancer on fluorouracil, } \\
\text { irinotecan, and bevacizumab }\end{array}$ & Surgical tissues, blood & Type b & $\begin{array}{l}\text { Ampicillin for } 51 \text { days; multiple debridements } \\
\text { (at least 2); recovery }\end{array}$ \\
\hline Robinson et al. (2010) & $\begin{array}{l}\text { 17-year-old female; right } \\
\text { arm and thigh }\end{array}$ & $\begin{array}{l}\text { SLE on mycophenolate and } \\
\text { hydroxychloroquine; } \\
\text { hypocomplementaemia } \\
\left(\mathrm{C} 3,18 \mathrm{mg} \mathrm{dl}^{-1} \text {; }\right. \\
\left.\text { C4, } 4 \mathrm{mg} \mathrm{dl}^{-1}\right)\end{array}$ & Surgical tissues, blood & Type e & $\begin{array}{l}\text { Vancomycin, nafcillin, clindamycin, and } \\
\text { cefotaxime for } 20 \mathrm{~h} \text {; one debridement; death } \\
\text { within } 20 \mathrm{~h} \text { of admission }\end{array}$ \\
\hline Chalmers (2010) & 64-year-old female; epiglottis & None & $\begin{array}{l}\text { Surgical tissues, blood, } \\
\text { epiglottis }\end{array}$ & Type b & $\begin{array}{l}\text { Ceftriaxone, clindamycin and metronidazole } \\
\text { changed to rifampicin for } 4 \text { days } \\
\text { post-debridement; one debridement; recovery }\end{array}$ \\
\hline Lee \& Ip (2010) & $\begin{array}{l}\text { 44-year-old male; right } \\
\text { buttock }\end{array}$ & $\begin{array}{l}\text { Intramuscular injections of } \\
\text { paracetamol to right buttock }\end{array}$ & Surgical tissues & Type b & $\begin{array}{l}\text { Intravenous ceftazidime and ciprofloxacin and } \\
\text { oral clarithromycin transitioned to ceftriaxone } \\
\text { (duration unknown); multiple debridements } \\
(n=5) \text {; recovery }\end{array}$ \\
\hline Saito et al. (2009) & 81-year-old female; left leg & Diabetes mellitus & Surgical tissues, blood & Type b & $\begin{array}{l}\text { Meropenem and minocycline for } 3 \text { days then } \\
\text { cefotaxime for } 28 \text { days; one debridement; } \\
\text { recovery }\end{array}$ \\
\hline McLellan et al. (2008) & 65-year-old male; left leg & Alcohol abuse & $\begin{array}{l}\text { Surgical tissues, left leg } \\
\text { wound swabs, } \\
\text { blood }\end{array}$ & Type f & $\begin{array}{l}\text { Benzylpenicillin, clindamycin, metronidazole } \\
\text { and cefuroxime, then meropenem added; one } \\
\text { debridement; death within } 14 \mathrm{~h} \text { of admission }\end{array}$ \\
\hline Stumvoll \& Fritsche (1997) & $\begin{array}{l}\text { 45-year-old male; right gluteus } \\
\text { and thigh }\end{array}$ & $\begin{array}{l}\text { Diabetes mellitus (haemoglobin } \\
\text { alc } 10 \% \text { ) }\end{array}$ & $\begin{array}{l}\text { Surgical tissues, } \\
\text { tracheal aspirate }\end{array}$ & Non-typable & $\begin{array}{l}\text { Amoxicillin-clavulanate, gentamicin, and } \\
\text { metronidazole followed by 'cephalosporin', } \\
\text { then ciprofloxacin for } 14 \text { days after } \\
\text { debridement; multiple debridements (daily); } \\
\text { recovery }\end{array}$ \\
\hline Collette et al. (1987) & $\begin{array}{l}\text { 13-month-old female; right } \\
\text { foot }\end{array}$ & $\begin{array}{l}\text { None; immunizations up to } \\
\text { date }\end{array}$ & Surgical tissues; blood & Type b & $\begin{array}{l}\text { Cefuroxime changed to oxacillin, } \\
\text { chloramphenicol and metronidazole, total of } \\
14 \text { days; multiple debridements (daily); } \\
\text { recovery }\end{array}$ \\
\hline
\end{tabular}


Patients with immunoglobulin deficiency are known to be at increased risk of a wide variety of infections with $H$. influenzae (Oksenhendler et al., 2008). Moreover, there is evidence that invasive infections such as meningitis, bacteraemia and peritonitis are more common in this patient population (Martinot et al., 2014). The patient in our study did not have known immunoglobulin deficiency.

H. influenzae may be either encapsulated (typable) or unencapsulated (non-typable). There are six encapsulated serotypes designated $\mathrm{a}-\mathrm{f}$ due to their distinct capsular polysaccharides. Hib has long been understood as the most virulent, capable of invasive disease and haematological dissemination by virtue of its polyribitol ribose phosphateladen capsule. Non-typable strains commonly cause upper respiratory infections in children and adults. With the introduction of the Hib vaccine in the late 1980s, the rates of invasive infections due to Hib as well as rates of nasopharyngeal colonization have dramatically decreased (Agrawal \& Murphy, 2011), through induction of both polyribitol ribose phosphate-targeting serum antibodies in vaccinated individuals and herd immunity in the unvaccinated. In the post-vaccine era, there is evidence that serotype switching, to a predominance of non-serotype b $\mathrm{H}$. influenzae, has occurred. In a population-based study of 91 cases of invasive disease with $H$. influenzae serotype a (Hia) was the most common serotype. The incidence of Hia-related disease increased over the 11-year study period, whilst that of Hib remained stable (Bender et al., 2010). In a retrospective study of 410 isolates of $H$. influenzae from blood and cerebrospinal fluid obtained over 12 years in Sweden, there was a significant increase over time in invasive disease caused by both non-typable $H$. influenzae and encapsulated strain type $\mathrm{f}$, measured as days of hospitalization per 100000 individuals. Only $7 \%$ of isolates in this study were identified as Hib (Resman et al., 2011). Other epidemiological reports have suggested that the shift has been predominantly towards non-typable strains (Agrawal \& Murphy, 2011). Among the previously reported cases of necrotizing fasciitis secondary to $H$. influenzae, five were Hib (Chalmers, 2010; Collette et al., 1987; Lee \& Ip, 2010; Saito et al., 2009; Ugai et al., 2014), one was Hie (Robinson et al., 2010), one was serotype f (Hif) (McLellan et al., 2008) and one was non-typable (Stumvoll \& Fritsche, 1997).

Most of the data on Hie comes from published case reports, leaving the specific manifestations of this pathogen largely uncharacterized. Invasive disease secondary to Hie including meningitis (Buck \& Douglas, 1976; Cerquetti et al., 2004; Schlossberg \& Crist, 1985) and sepsis (Cawthern et al., 1978; Schlossberg \& Crist, 1985) have been described. In a retrospective case series of 26 cases of infections with Hie, $61.5 \%$ were recovered from patients older than 16 years (Campos et al., 2003). Most presented with respiratory infections (54\%) and two had developed with invasive disease (sepsis and meningitis) but none had necrotizing fasciitis. In this series, Hie was strongly clonal and highly resistant to ampicillin (Campos et al., 2003). A study of laboratoryconfirmed Hif and Hie infections diagnosed during
2009-2010 in England and Wales found a $7.4 \%$ increase in the incidence of Hie (Ladhani et al., 2010). One case was described as a 'cellulitis' but none was described as necrotizing fasciitis. We found only one other case of necrotizing fasciitis in the literature caused by Hie (Robinson et al., 2010). Interestingly, both our patient and the patient described by Robinson et al. (2010) had SLE and hypocomplementaemia and were receiving mycophenolate and hydroxychloroquine.

The current case highlights the continued clinical importance of $H$. influenzae as a cause of invasive disease, including necrotizing fasciitis, particularly in immunocompromised individuals. This infection required multiple surgical debridements and pathogen-guided antimicrobial therapy to achieve a cure. Moreover, this case provides an additional example of invasive infection caused by Hie, supporting the emerging concern of invasive disease with non-b serotypes in the era of universal childhood Hib vaccination.

\section{Acknowledgements}

All authors contributed significantly to this work. The authors have no conflicts of interest.

\section{References}

Agrawal, A. \& Murphy, T. F. (2011). Haemophilus influenzae infections in the H. influenzae type b conjugate vaccine era. J Clin Microbiol 49, 3728-3732.

Bahu, S. J., Shibuya, T. Y., Meleca, R. J., Mathog, R. H., Yoo, G. H., Stachler, R. J. \& Tyburski, J. G. (2001). Craniocervical necrotizing fasciitis: an 11-year experience. Otolaryngolog Head Neck Surg 125, 245-252.

Bender, J. M., Cox, C. M., Mottice, S., She, R. C., Korgenski, K., Daly, J. A. \& Pavia, A. T. (2010). Invasive Haemophilus influenzae disease in Utah children: an 11-year population-based study in the era of conjugate vaccine. Clin Infect Dis 50, e41-e46.

Buck, L. L. \& Douglas, G. W. (1976). Meningitis due to Haemophilus influenzae type e. J Clin Microbiol 4, 381.

Campos, J., Román, F., Pérez-Vázquez, M., Oteo, J., Aracil, B., Cercenado, E. \& Spanish Study Group for Haemophilus influenzae Type E (2003). Infections due to Haemophilus influenzae serotype e: microbiological, clinical, and epidemiological features. Clin Infect Dis 37, 841-845.

Cawthern, T. H., Marraro, R. V. \& McCleskey, F. K. (1978). Bacteremia associated with Haemophilus influenzae type e biotype 4. J Clin Microbiol 7, 1-2.

Cerquetti, M., Ciofi degli Atti, M. L., Cardines, R., Giufre, M., Romano, A. \& Mastrantonio, P. (2004). Haemophilus influenzae serotype e meningitis in an infant. Clin Infect Dis 38, 1041.

Chalmers, C. (2010). Necrotising fasciitis complicating Haemophilus influenzae type b epiglottitis in an adult. J Laryngol Otol 124, 807-809.

Collette, C. J., Southerland, D. \& Corrall, C. J. (1987). Necrotizing fasciitis associated with Haemophilus influenzae type b. Am J Dis Child 141, 1146-1148.

Ladhani, S., Heath, P. T., Slack, M. P., Mclntyre, P. B., Diez-Domingo, J., Campos, J., Dagan, R., Ramsay, M. E. \& Participants of the European Union Invasive Bacterial Infections Surveillance Network (2010). Haemophilus influenzae serotype $b$ conjugate vaccine failure in twelve countries with established national childhood immunization programmes. Clin Microbiol Infect 16, 948-954. 
Lee, E. Y. \& Ip, W. Y. (2010). Necrotizing fasciitis of the extremity caused by Haemophilus influenzae serotype b in a healthy adult. Clin Orthop Relat Res 468, 1436-1439.

Martinot, M., Oswald, L., Parisi, E., Etienne, E., Argy, N., Grawey, I., DeBriel, D., Zadeh, M. M., Federici, L. \& other authors (2014). Immunoglobulin deficiency in patients with Streptococcus pneumoniae or Haemophilus influenzae invasive infections. Int $J$ Infect Dis 19, 79-84.

Mathieu, D., Neviere, R., Teillon, C., Chagnon, J. L., Lebleu, N. \& Wattel, F. (1995). Cervical necrotizing fasciitis: clinical manifestations and management. Clin Infect Dis 21, 51-56.

McLellan, E., Suvarna, K. \& Townsend, R. (2008). Fatal necrotizing fasciitis caused by Haemophilus influenzae serotype f. J Med Microbiol 57, 249-251.

Oksenhendler, E., Gérard, L., Fieschi, C., Malphettes, M., Mouillot, G., Jaussaud, R., Viallard, J. F., Gardembas, M., Galicier, L. \& other authors (2008). Infections in 252 patients with common variable immunodeficiency. Clin Infect Dis 46, 1547-1554.

Resman, F., Ristovski, M., Ahl, J., Forsgren, A., Gilsdorf, J. R., Jasir, A., Kaijser, B., Kronvall, G. \& Riesbeck, K. (2011). Invasive disease caused by Haemophilus influenzae in Sweden 1997-2009; evidence of increasing incidence and clinical burden of non-type b strains. Clin Microbiol Infect 17, 1638-1645.
Robinson, A. B., DeWitt, E. M., Schanberg, L. E. \& Moody, M. A. (2010). Necrotizing fasciitis caused by Haemophilus influenzae typee in a 17-year-old girl with systemic lupus erythematosus. J Clin Rheumatol 16, 49-50.

Saito, T., Matsunaga, H., Matsumura, Y., Segawa, H., Takakura, S., Nagao, M., linuma, Y., Miyachi, Y. \& Ichiyama, S. (2009). Necrotizing fasciitis caused by Haemophilus influenzae type b in an elderly patient. J Clin Microbiol 47, 852-854.

Schlossberg, D. \& Crist, A. E. Jr (1985). Meningitis and septicemia due to Haemophilus influenzae serotype e, biotype IV. Diagn Microbiol Infect Dis 3, 73-75.

Stumvoll, M. \& Fritsche, A. (1997). Necrotizing fasciitis caused by unencapsulated Haemophilus influenzae. Clin Infect Dis 25, 327.

Tedesco, F. (2008). Inherited complement deficiencies and bacterial infections. Vaccine 26 (Suppl. 8), I3-I8.

Ugai, T., Norizuki, M., Mikawa, T., Ohji, G. \& Yaegashi, M. (2014). Necrotizing fasciitis caused by Haemophilus influenzae type b in a patient with rectal cancer treated with combined bevacizumab and chemotherapy: a case report. BMC Infect Dis 14, 198.

Winkelstein, J. A. \& Moxon, E. R. (1992). The role of complement in the host's defense against Haemophilus influenzae. J Infect Dis 165 (Suppl. 1), S62-S65. 